\title{
EFFECTS OF AMRINONE ON THE TRACHEAL MUSCULATURE AND VASCULATURE OF THE DOG
}

\author{
Masahiko MARUYAMA, Keisuke SATOH and Norio TAIRA \\ Department of Pharmacology. Tohoku University School of Medicino. \\ Sendai 980 , Japan
}

Accepted September 9, 1981

Amrinone is a newly developed positive inotropic drug $(1,2)$. The mechanism of its positive inotropic action has not been elucidated since. Inhibition of $\mathrm{Na}-\mathrm{K}$ dependent ATPase (1) and an increase in cyclic AMP level either via $\beta$-adrenoceptor stimulation (1) or due to inhibition of cyclic AMP phosphodiesterase in cardiac muscle (3) have been considered to be unlikely. Recently, however, amrinone was reported to increase intracellular cyclic AMP levels in the rabbit aorta during a time period when the contractile response to noradrenaline was inhibited (4). Such observations suggested the possible involvement of cyclic AMP in the action of amrinone on smooth muscle (4). The present experiments analyzed how amrinone affects tracheal smooth muscle and vascular smooth muscle of the dog trachea, since an increase in intracellular cyclic AMP level in tracheal smooth muscle leads to relaxation as in the case of vascular smooth muscle (5).

In 10 mongrel dogs, weighing 10 to $15 \mathrm{~kg}$. anesthetized with sodium pentobarbital (30 $\mathrm{mg} / \mathrm{kg}$, i.v.) and given sodium heparin (500 units $/ \mathrm{kg}$. i.v.), the tracheal vascular bed was perfused in situ with arterial blood through both cannulated cranial thyroid arteries. Perfusion pressure was kept constant at a value slightly higher than the mean systemic arterial pressure. Blood flow through the arteries (tracheal blood flow) was measured with an electromagnetic flowmeter. The trachea was intubated by a tracheal tube with a water-filled cuff attached. Hydraulic pressure in the cuff was measured with a pressure transducer as intraluminal pressure of the trachea (tracheal tone). Details of the methods were described previously (6). Tracheal tone was maintained elevated by intra-arterial injections of $3 \mu \mathrm{g}$ of neostigmine methyl sulfate (Shionogi, in ampoule) at about $30 \mathrm{~min}$ intervals. (-)-Isoproterenol hydrochloride (Sigma), amrinone (SterlingWinthrop) and ( \pm )-propranolol hydrochloride (ICI) were used. Isoproterenol and propranolol were dissolved in $0.9 \%$ saline. Amrinone was dissolved at a concentration of $10 \mathrm{mg} / \mathrm{ml}$, in $0.1 \mathrm{~N}$ lactic acid and was diluted with saline to the desired concentrations. Doses of isoproterenol and propranolol refer to their bases. All drug solutions in a volume of $30 \mu \mathrm{l}$ were injected intraarterially in $4 \mathrm{sec}$. Values in the text are expressed in terms of mean \pm S.E. (unless otherwise stated). Dose-response curves were treated as linear regressions and parallelism of the curves was analyzed.

The mean systemic arterial pressure was $118 \pm 7 \mathrm{mmHg}(n=10)$. The mean tracheal blood flow was $6 \pm 1 \mathrm{ml} / \mathrm{min}$ at the mean perfusion pressure of $136 \pm 20$ (S.D.) $\mathrm{mm}$ $\mathrm{Hg}(n=10)$. The mean basal intraluminal pressure elevated by repeated injections of $3 \mu \mathrm{g}$ of neostigmine methyl sulfate was $67 \pm 3 \mathrm{~cm} \mathrm{H}_{2} \mathrm{O}$.

Amrinone ( 3 to $300 \mu \mathrm{g}$, i.a.) and isoproterenol $(0.03$ to $0.3 \mu \mathrm{g}$, i.a.) increased tracheal blood flow (tracheal vasodilatation) 


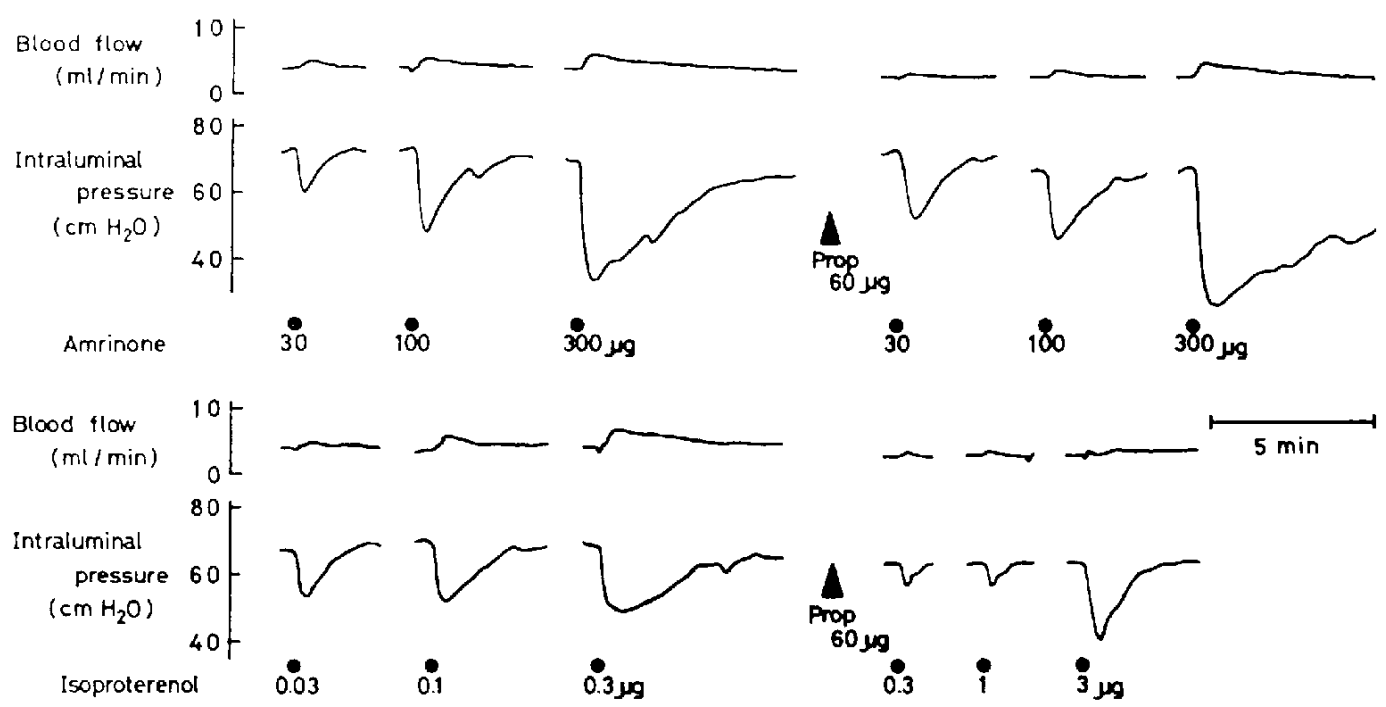

Fig. 1. Responses of the tracheal vascular bed (blood flow) and musculature (intraluminal pressure) to intra-arterial amrinone and isoproterenol, and modification by intra-arterial injection of propranolol (Prop).

and decreased tracheal tone (tracheal dilatation) in a dose-dependent manner (Fig. 1), and at high doses, the two drugs produced maximal tracheal dilatation. However, even in doses producing maximal tracheal dilatation, amrinone, unlike isoproterenol. failed to produce maximal tracheal vasodilatation. Dose-response curves to amrinone for tracheal vasodilatation and tracheal dilatation were parallel $(p<0.05)$ to the corresponding curves to isoproterenol (Fig. 2). In producing tracheal vasodilatation and tracheal dilatation, amrinone was about 22,000 and 1.200 times less potent than isoproterenol on a weight basis, respectively. Selectivity of each drug for tracheal musculature against vasculature was determined from the ratio of the dose required to increase tracheal blood flow by $3 \mathrm{ml} / \mathrm{min}$ to that required to decrease intraluminal pressure by $15 \mathrm{~cm} \mathrm{H}_{2} \mathrm{O}$. When the selectivity of isoproterenol was assigned to be unity. that of amrinone was 14; amrinone was about 14 times more selective for tracheal musculature than for tracheal vasculature as compared with isoproterenol.
Propranolol (60 $\mu \mathrm{g}$, i.a.) shifted the doseresponse curves to isoproterenol for tracheal vasodilatation to the right by about $1.4 \mathrm{log}$ units and that for tracheal dilatation by about $1.2 \mathrm{log}$ units. This dose of propranolol. however, failed to antagonize both tracheal vasodilatation and tracheal dilatation produced by amrinone (Fig. 2).

In the present experiments, like isoproterenol, amrinone produced tracheal dilatation and tracheal vasodilatation. However, the actions of amrinone, unlike those of isoproterenol, were not modified by propranolol as reported by previous investigators $(1,4)$. In producing tracheal vasodilatation amrinone was about 25,000 times less potent than isoproterenol on a molar basis. A similar value has been obtained for vasodilator effects in isolated, blood-perfused dog papillary muscle preparations (7). In the same preparations, amrinone was about 100.000 times less potent than isoproterenol on a molar basis in producing a positive intropic action (7). Interestingly, in the present experiments, in producing tracheal dilatation amrinone was about 1,400 times 

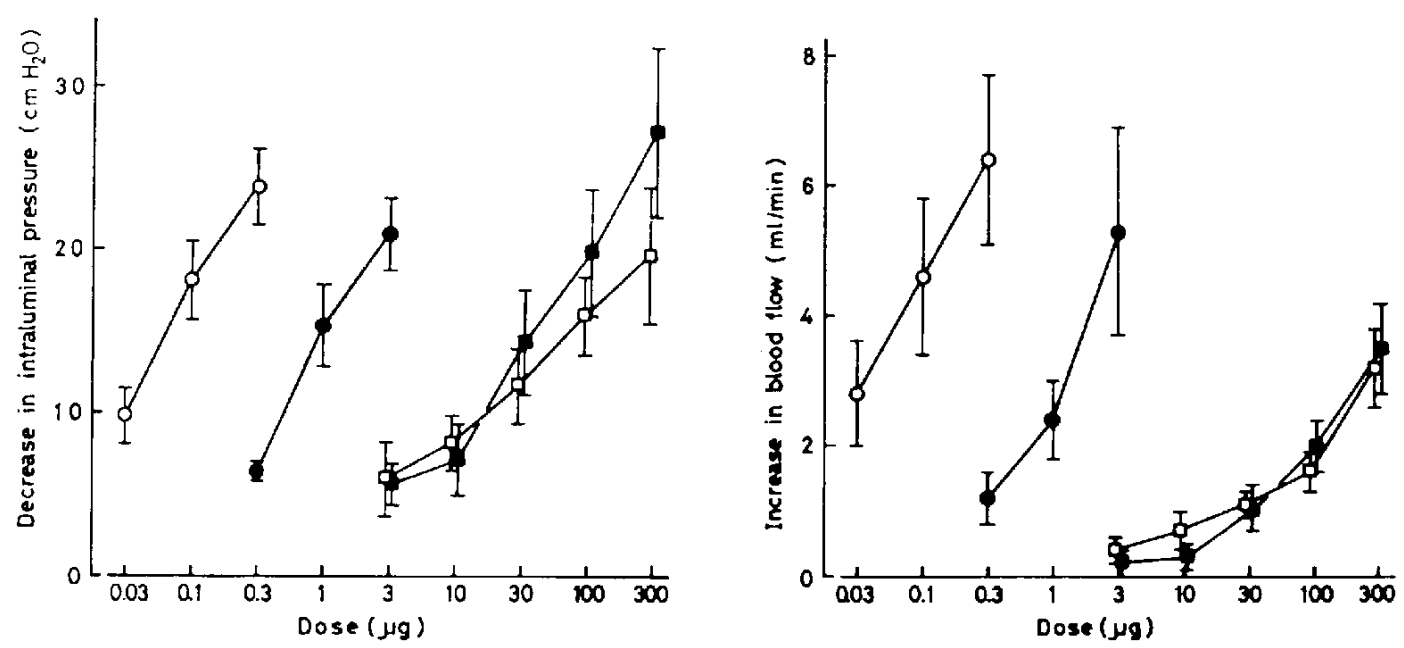

Fig. 2. Left panel: Dose-response curves for decrease in tracheal intraluminal pressure to isoproterenol before (O) and after (O) propranolol (60 \%g, i.a.) and to amrinone before ( $\square$ ) and after $(\square)$ propranolol (60 kg. i.a.). Right pancl: Similar doso-rcsponse curves. but for increase in tracheal blood flow. Each point represents the mean valuc of $5-6$ observations on 5-6 dogs, and vertical bars show the S.E.

less potent than isoproterenol on a molar basis. Thus, amrinone can be characterized as a tracheo-bronchodilator, rather than a positive inotropic drug.

In having tracheodilator, vasodilator and positive inotropic actions independent of a $\beta$-adrenoceptor mechanism amrinone resembles theophylline and papaverine. The present results thus tempt us to speculate that an increase in intracellular cyclic AMP level may underlie these actions of amrinone. as suggested by Meisheri et al. (4) about the inhibitory action of amrinone on the contractile response of the rabbit aorta to noradrenaline. The high selectivity of amrinone for tracheal musculature against vasculature implies that amrinone may have potential use for treatment of bronchial asthma.

Acknowledgements: We are grateful to the Sterling-Winthrop Research Institute. New York, U.S.A. for generously supplying amrinone. This work was supported by a Grant-in-Aid for Encouragement of Young Scientists (No. 56770110) form the Ministry of Education, Science and Culture, Japan.

\section{REFERENCES}

1) Farah, A.E. and Alousi, A.A.: New cardiac agents: A search for digitalis substitute. Life Sci. 22, 1139-1148 (1978)

2) Benotti, J.R., Grossman, W., Braunwald, E., Davolos, D.D. and Alousi, A.A.: Hemodynamic assessment of amrinone: A new inotropic agent. New Eng. J. Med. 299, 1373-1377 (1978)

3) Alousi, A.A., Farah, A.E., Lesher, G.Y. and Opalka, C.J., Jr.: Cardiotonic activity of amrinone-Win 40680 [5-amino-3,4'bipyridin-6 (1H)-one]. Circ. Res. 45, 666-677 (1979)

4) Meisheri, K.D., Palmer, R.F. and van Breemen, C.: The effects of amrinone on contractility. $\mathrm{Ca}^{2+}$ uptake and CAMP in smooth muscle. Europ. J. Pharmacol. 61, 159-165 (1980)

5) Bär, H.-P.: Cyclic nucleotides and smooth muscle. Adv. Cyclic Nucleotide Res. 4, 195-237 (1974)

6) Himori, N. and Taira, N.: A method for recording smooth muscle and vascular responses of the blood-perfused dog trachea in situ. Brit. J. Pharmacol. 56, 293-299 (1976)

7) Yamashita, S. and Taira, N.: Cardiac effects of amrinone as assessed in isolated. bloodperfused canine heart preparations. Abstracts of Papers presented at the 8 th International Congress of Pharmacology (Tokyo). (1981) 